\title{
Chlamydia trachomatis Infections: Implications for Pregnant Adolescents and Their Infants
}

\author{
Marlene Melzer-Lange, Laurie Good, and Halim Hennes \\ Department of Pediatrics, Medical College of Wisconsin (M.M.-L., H.H.), and Teen Pregnancy \\ Service of Milwaukee (L.G.), Milwaukee, WI
}

\begin{abstract}
Objective: Chlamydia trachomatis infections are common in pregnant adolescents. Previous studies have shown that treating pregnant women of all ages with erythromycin prevents transmission of this infection to their infants. However, there are no published studies on the efficacy of aggressive screening and treatment of $C$. trachomatis in pregnant adolescents. This study was undertaken to determine if aggressive screening for $C$. trachomatis in pregnant adolescents and early treatment with erythromycin can prevent complications in their newborn infants.

Methods: A group of pregnant adolescents enrolled at Teen Pregnancy Service of Milwaukee was evaluated prospectively for the presence of $C$. trachomatis infection. Screening was performed during the 1st and 3rd trimesters by enzyme immunoassay. Adolescents with positive enzyme immunoassays for Chlamydia were treated with erythromycin for 10 days. Those with negative enzyme immunoassays were enrolled as controls. All infants born to adolescents in both groups were followed for episodes of conjunctivitis, pneumonia, and wheezing during their 1st year of life.

Results: Ninety mother/infant pairs were followed during the study period. Twenty-eight mothers $(31 \%)$ had positive enzyme assay tests and all received erythromycin therapy. Nasopharyngeal cultures were obtained from $60(67 \%)$ infants; all were negative. There were no significant differences in general characteristics, development of conjunctivitis (relative risk 1.27), wheezing (relative risk 0.91), or pneumonia (relative risk 1.12) between infants born to adolescents in either group.

Conclusions: We conclude that aggressive screening and treatment of $C$. trachomatis infection in pregnant adolescents may prevent complications in their offspring. $\quad$ @ 1994 Wiley-Liss, Inc.
\end{abstract}

Adolescent pregnancy, sexually transmitted disease, prenatal care

C hlamydia trachomatis is a common sexually transmitted infection among pregnant adolescents; the infection rate ranges from 14 to $27 \% .^{1-3}$ Infections during pregnancy are often transmitted to the infant at the time of delivery. ${ }^{4-6}$ Rates of transmission vary from 50 to $75 \%$ of infants born to infected mothers. Previous studies in women of all childbearing ages have shown that the use of erythromycin during pregnancy diminishes $C$. tra- chomatis transmission to the infant and prevents complications such as conjunctivitis and pneumonia. ${ }^{7,8}$ Adolescents are particularly at risk for poor compliance with prenatal care and medication administration and for reinfection with C. trachomatis. ${ }^{9-11}$ There are no studies that have examined the effectiveness of erythromycin during pregnancy in a purely adolescent population.

This prospective cohort study was undertaken to

Address correspondence/reprint requests to Dr. Marlene Melzer-Lange, Department of Pediatrics, Children's Hospital of Wisconsin, 9000 West Wisconsin Avenue, MS \#677, Milwaukee, WI 53226.

Presented in part at the 32nd Annual Meeting of the Ambulatory Pediatric Association, Baltimore, MD, May 1992. 
evaluate whether infants born to adolescents treated for $C$. trachomatis with erythromycin during pregnancy were at increased risk for the complications of wheezing, conjunctivitis, and pneumonia compared with infants born to mothers who were negative for $C$. trachomatis during pregnancy.

\section{SUBJECTS AND METHODS}

During a 12-month period, all pregnant adolescents presenting for prenatal care at Teen Pregnancy Service of Milwaukee were screened for $C$. trachomatis infection. An enzyme immunoassay test (Chlamydiazyme $^{\mathrm{TM}}$, Abbott Laboratories, North Chicago, IL) was performed at intake, 36 weeks gestation, and 6-8 weeks postpartum. A mother was considered $C$. trachomatis positive if a chlamydial enzyme immunoassay was positive during pregnancy. Adolescents with $C$. trachomatis were treated within 1 week of testing with erythromycin base, $500 \mathrm{mg}$ t.i.d. for 10 days, and retested within 1 month. C. trachomatis infections detected at any time during pregnancy were also treated with erythromycin at the same dose. Prenatal testing, treatment, and instruction regarding chlamydial infection were provided to all patients by a certified nurse-midwife on a one-to-one basis. A multidisciplinary program supported all pregnant teens throughout pregnancy with prenatal education classes, social worker intervention, nutritional counseling through the Women Infant Children Nutrition Site (WIC), and group counseling sessions at the same site. Sexual partners were referred for treatment. At the time of delivery, all infants received erythromycin ophthalmic ointment prophylaxis. Demographic data and smoking history were obtained from each patient.

Infants of both C. trachomatis-positive and -negative mothers were followed prospectively for 1 year for episodes of conjunctivitis, pneumonia, and wheezing. Visits were scheduled monthly for the 1st 6 months of the infant's life and then every other month until the 1st birthday. Conjunctivitis was defined as erythema with drainage of 1 or both eyes. Pneumonia was defined as rales in conjunction with abnormal chest X-ray. Wheezing was defined as tachypnea associated with expiratory wheezes on auscultation. Episodes were identified during either well-baby visits or sick visits at the clinic or emergency department; history of eye drainage or respiratory distress was requested from
TABLE I. General characteristics

\begin{tabular}{lccc}
\hline & $\begin{array}{c}\text { C. trachomatis } \\
\text { positive } \\
(\mathrm{N}=28)\end{array}$ & $\begin{array}{c}\text { C. trachomatis } \\
\text { negative } \\
(\mathrm{N}=62)\end{array}$ & $P$ \\
\hline Race & & & \\
Black & $23(82 \%)$ & $46(74 \%)$ & \\
White & $3(11 \%)$ & $6(10 \%)$ & \\
Other & $2(7 \%)$ & $10(16 \%)$ & \\
Maternal age (years) & $16.8 \pm 0.3$ & $17.3 \pm 0.2$ & $0.2^{\mathrm{c}}$ \\
Infant weight (g) & $3,149 \pm 114$ & $3,194 \pm 75$ & $0.7^{\mathrm{c}}$ \\
No. of smokers & 8 & 17 & $0.9^{\mathrm{d}}$ \\
No. of clinic visits & 10 & 11 & $0.3^{\mathrm{c}}$ \\
\hline a'Mean \pm SEM. & & & \\
bTotal number of smokers in each group. & & \\
'Student's t-test. & & & \\
'Chi-squared test. & & &
\end{tabular}

care givers at each clinic visit. Access to all medical records for visits was assured since patients were enrolled in a managed-care plan. Nasopharyngeal swabs of infants in both groups were collected between 1 and 6 months of age on Chlamydia Transwab $^{\circledR}$ (Microdiagnostics Corp., Cleveland, OH) and sent directly to the laboratory for $\mathrm{McC}$ oy cell culture during well-child visits. Infants with pneumonia or conjunctivitis were also cultured for $C$. trachomatis at the time of their acute illness. Data were analyzed using the Student's t-test, chi-squared test, and Mann-Whitney rank test. Relative risks, with $95 \%$ confidence interval, were calculated for outcome variables.

\section{RESULTS}

One hundred ten mother/infant pairs were enrolled and followed during the study period. Twenty pairs were excluded from the study due to change in care providers and loss of follow-up of the infants. There were no differences in the excluded group and the group studied by race, smoking, age, birth weight, or $C$. trachomatis status. Twenty-eight mothers were $C$. trachomatis positive and 62 were C. trachomatis negative. Estimated gestational age at intake was 17.2 weeks (range 10-27 weeks) for C. trachomatis-positive mothers and 16.9 weeks (range 11-30 weeks) for C. trachomatis-negative mothers. The demographic characteristics for the 2 groups are summarized in Table 1. There were no significant differences for maternal age, race, birth weight, or history of maternal smoking. Eighty-eight of 90 (98\%) mothers were eligible for Medicaid. 
TABLE 2. Mothers with evidence of $C$. trachomatis during pregnancy ${ }^{a}$

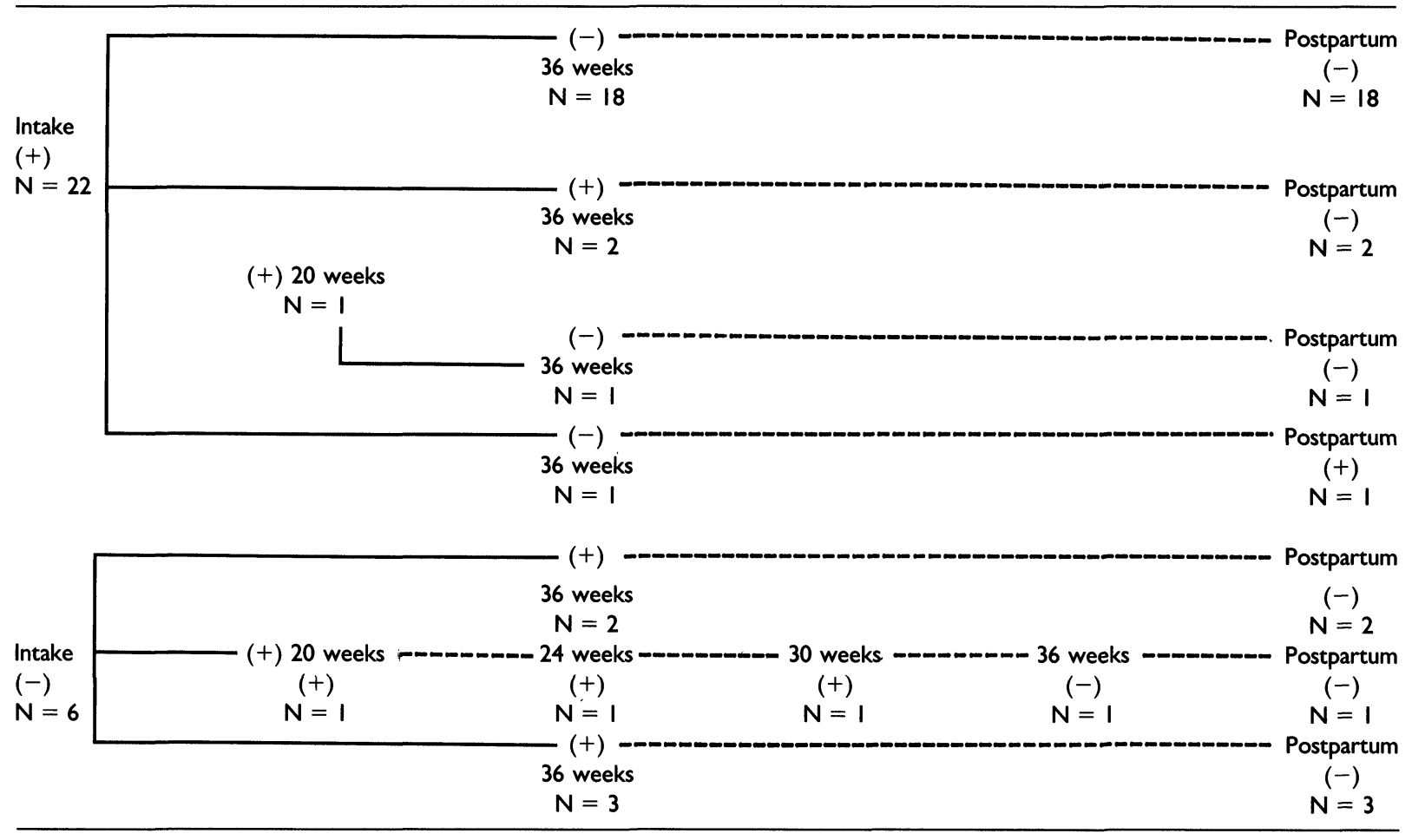

${ }^{a}+=$ positive chlamydial enzyme immunoassay.

Chlamydial enzyme immunoassay was positive in 22 mothers at intake. Six additional mothers became positive for $C$. trachomatis during pregnancy. Table 2 summarizes the cervical chlamydial enzyme immunoassay results throughout pregnancy and postpartum.

Of the 28 infants born to mothers treated for $C$. trachomatis, 21 (75\%) were cultured for C. trachomatis; all were negative. Forty (64\%) infants born to $C$. trachomatis-negative mothers were cultured; all were culture negative. There were no positive cultures for $C$. trachomatis during episodes of conjunctivitis or pneumonia.

Of the 28 infants born to mothers treated for $C$. trachomatis, 12 developed conjunctivitis, 2 developed pneumonia, and 12 developed wheezing. Of the 62 infants born to $C$. trachomatis-negative mothers, 23 developed conjunctivitis, 6 developed pneumonia, and 28 developed wheezing. There was no statistical difference between the 2 groups in the number of episodes of upper respiratory infection, conjunctivitis, pneumonia, or wheezing by the Mann-Whitney rank test. Relative risk for conjunctivitis was 1.27 , for pneumonia was 1.12 , and for wheezing was 0.91 between the 2 groups ( $\mathrm{Ta}$ ble 3).

\section{DISCUSSION}

The literature provides little data on the compliance of treatment of $C$. trachomatis among pregnant adolescents and the outcome of their infants. Although compliance may be a problem for adolescents, we found that most mothers treated early in their pregnancy for $C$. trachomatis remained disease-free throughout pregnancy. Infants born to mothers treated for $C$. trachomatis showed no evidence of nasopharyngeal carriage nor did they have conjunctivitis, pneumonia, or wheezing more frequently than infants born to $C$. trachomatis-negative mothers.

Adolescents, especially during pregnancy, are at greater risk for $C$. trachomatis infections than adult women. In our study, $31 \%$ of pregnant adolescents had C. trachomatis infection. Earlier studies ${ }^{1-3}$ have reported an infection rate between 14 and $27 \%$. In another study conducted in Milwaukee, $11 \%$ of pregnant adult women (mean age 24 years) were found to be infected with $C$. trachomatis. ${ }^{12}$ Factors 
TABLE 3. Relationship between disease outcome and maternal $C$. trachomatis results

\begin{tabular}{lcccc}
\hline & $\begin{array}{c}\text { C. trachomatis positive } \\
(\mathrm{N}=28)\end{array}$ & $\begin{array}{c}\text { C. trachomatis negative } \\
(\mathrm{N}=62)\end{array}$ & $\begin{array}{c}\text { Relative } \\
\text { risk }\end{array}$ & $\begin{array}{c}95 \% \\
\text { confidence } \\
\text { interval }\end{array}$ \\
\hline Conjunctivitis & 12 & 23 & 1.27 & $0.52,3.06$ \\
No conjunctivitis & 16 & 39 & & 0.91 \\
Wheezing & 12 & 28 & $0.36,2.21$ \\
No wheezing & 16 & 34 & 1.12 & $0.19,6.4$ \\
Pneumonia & 2 & 6 & 56 & \\
No pneumonia & 26 & & & \\
\hline
\end{tabular}

which account for the high prevalence of $C$. trachomatis in adolescents may include increased sexual activity at an earlier age and multiple sexual partners. Furthermore, pregnant adolescents may also be more likely than nonpregnant adolescents to be infected with C. trachomatis. In Brooklyn, NY, the rates were $16 \%$ in pregnant adolescents compared with $7.3 \%$ in nonpregnant adolescents. ${ }^{1}$ Chacko and Lovchik ${ }^{3}$ reported C. trachomatis rates of $27 \%$ in pregnant teens and $23 \%$ in nonpregnant teens.

The enzyme immunoassay method was used for the detection of $C$. trachomatis in the endocervical samples from the pregnant adolescents in this study. This method was selected because it is easy to transport and the results can be obtained within 1 day. Though culture by McCoy cell has been the gold standard for detection of chlamydial infection, the enzyme immunoassay has been shown to be appropriate for the screening of pregnant women and adolescents. Baselski et al. ${ }^{13}$ showed the enzyme immunoassay (Chlamydiazyme ${ }^{\mathrm{TM}}$ ) to have a sensitivity of 96.3 and a specificity of $92.9 \%$ compared with culture in a group of pregnant women. In a study of female adolescents, the enzyme immunoassay demonstrated a sensitivity of $100 \%$ and a specificity of $88 \% .^{14}$

The timing of $C$. trachomatis screening and erythromycin therapy during pregnancy is controversial. Schachter et al. ${ }^{7}$ treated pregnant patients at 36 weeks gestation in hopes of allowing a $C$. trachomatis-free genital tract at the time of delivery. Eight of 152 infants in that study were born while the mother was still taking erythromycin; 2 infants developed C. trachomatis infections. Other studies have examined the efficacy of erythromycin for treatment of $C$. trachomatis during pregnancy. ${ }^{15}$ However, the efficacy has not been examined in a purely adolescent population.

Compliance, risk-taking behavior, multiple sexual partners, and poor condom use are all factors that may make $C$. trachomatis treatment in pregnant adolescents problematic. We felt that an aggressive approach to the eradication of $C$. trachomatis during the entire gestation of an adolescent pregnancy is appropriate. In our study, pregnant adolescents were screened and treated at intake and again at 36 weeks gestation. Those adolescents who were positive for $C$. trachomatis at intake were aggressively treated and tests of cure were performed 2 weeks after completion of therapy. Although the use of erythromycin during pregnancy has been reported to be associated with poor compliance due to gastrointestinal side effects, ${ }^{16}$ the adolescents in our study reported completion of their erythromycin course on their follow-up visits. Early testing of pregnant teens permitted identification and treatment of sexual partners. Any adolescent who reported exposure during pregnancy to C. trachomatis from an infected partner was also retested and treated. In the present study, $82 \%$ of mothers treated for $C$. trachomatis during the early months of pregnancy remained negative throughout the pregnancy. With aggressive management, C. trachomatis was eradicated in an additional $14 \%$ of teens prior to delivery. Treatment initiated at detection of $C$. trachomatis at intake was successful in our study and most adolescents remained negative throughout their pregnancy. Patients negative at intake for $C$. trachomatis who became infected later in pregnancy were detected at testing at 36 weeks. 
Although current Centers for Disease Control ${ }^{17}$ recommendations advise testing and treatment at 36 weeks, our data suggest that evaluation should be done at both the prenatal intake and the $3 \mathrm{rd}$ trimester to control chlamydial infection in adolescents.

Another argument may be made to support the recommendation for chlamydial detection early in pregnancy. Recent studies have examined the relationship between $C$. trachomatis infection and premature labor and low birth weight. ${ }^{15,18}$ Ryan et al. ${ }^{19}$ showed birth weights of $<2,500 \mathrm{~g}$ in $19 \%$ of infants of untreated $C$. trachomatis-positive mothers vs. $11 \%$ of treated mothers. Others, ${ }^{20}$ however, suspect that mucopurulent cervicitis, unrelated to C. trachomatis infection, may be a predictor of adverse pregnancy outcome. Erythromycin therapy, unrelated to $C$. trachomatis infection, may also improve birth weights; McCormack et al. ${ }^{21}$ showed fewer low-birth-weight infants in a group of women infected with Ureaplasma urealyticum treated with erythromycin during the 3 rd trimester. In the present study, birth weights were not significantly different in the 2 groups of patients.

The compliance of the pregnant teens in our study was very good, with most presenting for care by 20 weeks gestation and most receiving 10-11 prenatal visits. Our program offers a multidisciplinary approach to teen pregnancy which includes medical, nutritional, prenatal education, pediatric (for siblings), and social services to the pregnant teen and her children at one inner-city site. Most patients are referred by other adolescents and may partly account for the initiation of care prior to the 3 rd trimester. We believe that the compliance of our pregnant adolescents may be better than what could be attained through prenatal care at a site not dedicated to the pregnant teen.

All cultures of infants for $C$. trachomatis were negative. Cell culture by $\mathrm{McC}$ oy cell technique was employed for detection of $C$. trachomatis in the infants since nonculture techniques have not been well studied in the infant population. Although no cultures in the study infants were positive for $C$. trachomatis, cultures obtained in the same manner were positive in another group of infants during the same time period.

Because cultures may be somewhat fastidious, infants were followed for symptoms that could be related to $C$. trachomatis infection. ${ }^{13}$ In examining conjunctivitis, pneumonia, and wheezing in the 2 groups, we found no differences in risk between infants born to mothers who were $C$. trachomatis negative and those born to mothers treated during pregnancy for $C$. trachomatis. Although C. trachomatis may be implicated in $1 / 3$ of infants with pneumonia, ${ }^{22}$ we found no increase in risk for pneumonia between the infants born to infected and noninfected mothers. Although $C$. trachomatis conjunctivitis has been found as often as $51 \%$ of the time in young infants with conjunctivitis, ${ }^{23}$ we found no increase in risk for conjunctivitis between infants born to infected and noninfected mothers.

Wheezing following $C$. trachomatis infection has been reported by several authors. ${ }^{24-26}$ Chronic $C$. trachomatis infections in infants have also been reported, some lasting over 2 years. ${ }^{27}$ No evidence of increased wheezing or chronic infection was found in infants in the group born to mothers treated for C. trachomatis.

The cost of screening and treatment $(\$ 84.00)$ of pregnant teens is far less than the cost of outpatient and in some cases inpatient care of infants with $C$. trachomatis pneumonia. If, as suspected, C. trachomatis contributes to long-term chronic respiratory disease in older children, further costs will ensue. $^{28}$

\section{CONCLUSIONS}

Our study demonstrates that screening and treatment for $C$. trachomatis in pregnant adolescents early and throughout pregnancy may be effective in eradicating infection and preventing infectious complications in their offspring. Further studies are needed to investigate the impact that $C$. trachomatis and erythromycin have on the incidence of low birth weight in adolescent pregnancies. Should the newer one-dose antibiotics such as azithromycin be found to be safe for use during pregnancy, treatment may become less complicated. Programs serving pregnant teens must target $C$. trachomatis as a common infection and offer early and persistent evaluation and treatment.

\section{ACKNOWLEDGMENTS}

We thank Margo Kinservik, P.N.P., and Carmen Otero, P.N.P., for their assistance in obtaining infant cultures and Frances Sommer for assistance in preparation of the manuscript. This project was 
supported in part by a grant from the Wisconsin Perinatal Foundation.

\section{REFERENCES}

1. Golden N, Hammerschlag M, Neuhoff S, Gleyzer A: Prevalence of Chlamydia trachomatis cervical infection in female adolescents. Am J Dis Child 138:562-564, 1984.

2. Hammerschlag MR, Cummings C, Roblin PM, Williams TH, Delke I: Efficacy of neonatal ocular prophylaxis for the prevention of chlamydial and gonococcal conjunctivitis. N Engl J Med 320:769-772, 1989.

3. Chacko MR, Lovchik JC: Chlamydia trachomatis infection in sexually active adlescents: Prevalence and risk factors. Pediatrics 73:836-840, 1984.

4. Frommell GT, Rothenberg R, Wang SP, McIntosh K: Chlamydia infection of mothers and infants. Pediatrics 95:28-32, 1979.

5. Hammerschlag MR, Anderka M, Semine DZ, McComb D, McCormack WM: Prospective study of maternal and infantile infection with Chlamydia trachomatis. Pediatrics 64:142-147, 1979.

6. Schachter J, Grossman M, Sweet RL, Holt J, Jordan C, Bishop E: Prospective study of perinatal transmission of Chlamydia trachomatis. JAMA 255:3374-3377, 1986.

7. Schachter J, Sweet RL, Grossman M, Landers D, Robbie $\mathrm{M}$, Bishop E: Experience with the routine use of erythromycin for chlamydial infections in pregnancy. $\mathrm{N}$ Engl J Med 314:276-279, 1986.

8. Heggie AD, Lumicao GG, Stuart LA, Gyves MT: Chlamydia trachomatis infection in mothers and infants. Am J Dis Child 135:507-511, 1981.

9. Bell TA, Farrow JA: Compliance with treatment for sexually transmitted diseases. Semin Adol Med 3:153-156, 1987.

10. Smith PB, Chacko MR, McGill L, Phillips LE: Sexually transmitted disease treatment and return for test of cure of adolescents in a family planning clinic. J Adol Health 12:49-52, 1991.

11. Katz BP, Zwickl BW, Caine VA, Jones RB: Compliance with antibiotic therapy for Chlamydia trachomatis and Neisseria gonorrhoeae. Sex Transm Dis 19:351-354, 1992.

12. Baumgardner DJ, Christopherson A, Momont S: Chlamydia in pregnant women: Southwestern Wisconsin. Wis Med J 88(9):12-15, 1989.

13. Baselski VS, McNeeley SG, Ryan G, Robison M: A comparison of nonculture-dependent methods for detection of Chlamydia trachomatis infections in pregnant women. Obstet Gynecol 70:47-52, 1987.
14. Krowchuk DP, Anglin TM, Lembo RM, Brown RF, Thomas F, Kumar ML: Use of enzyme immunoassay for the rapid diagnosis of Chlamydia trachomatis endocervical infection in female adolescents. J Adol Health Care 9:296300, 1988.

15. Sweet RL, Landers DV, Walker C, Schachter J: Chlamydia trachomatis infection and pregnancy outcome. Am J Obstet Gynecol 156:824-833, 1987.

16. Alger LS, Lovchik JC: Comparative efficacy of clindamycin versus erythromycin in eradication of antenatal C. trachomatis. Am J Obstet Gynecol 165:375-381, 1991.

17. Centers for Disease Control: Recommendations for the prevention and management of Chlamydia trachomatis infections. MMWR 42:1-39, 1993.

18. Cohen I, Veille JC, Calkins BM: Improved pregnancy outcome following successful treatment of chlamydial infection. JAMA 263:3160-3163, 1990.

19. Ryan GM, Abdella TN, McNeeley SG, Baselski VS, Drummond DE: Chlamydia trachomatis infection in pregnancy and effect of treatment on outcome. Am J Obstet Gynecol 162:34-39, 1990.

20. Nugent RP, Hillier SL: Mucopurulent cervicitis as a predictor of chlamydial infection and adverse pregnancy outcome. Sex Transm Dis 19:198-202, 1992.

21. McCormack WM, Rosner B, et al.: Effect on birth weight of erythromycin treatment of pregnant women. Obstet Gynecol 69:202-207, 1987.

22. Brasfield DM, Stagno S, Whitley RJ, Cloud G, Cassell G, Tiller RE: Infant pneumonitis associated with cytomegalovirus, Chlamydia, Pneumocystis, and Ureaplasma: Follow-up. Pediatrics 79:76-83, 1987.

23. Barry WC, Teare EL, Uttley AHC, et al.: Chlamydia trachomatis as a cause of neonatal conjunctivitis. Arch Dis Child 61:797-799, 1986.

24. Bavastrelli M, Midulla M, Rossi D, Salzano M: Chlamydia trachomatis infection in children with wheezing simulating asthma. Lancet 339:1174, 1992.

25. Carballal G, Mahony JB, Videla C, Cerqueiro C, Chernesky $\mathrm{M}$ : chlamydial antibodies in children with lower respiratory disease. Pediatr Infect Dis J 11:68-71, 1991.

26. Weiss SG, Newcomb RW, Beem MO: Pulmonary assessment of children after chlamydial pneumonia of infancy. J Pediatr 108:659-664, 1986.

27. Bell TA, Stamm WE, Wang SP, Kuo CC, Holmes KK, Grayston JT: Chronic Chlamydia trachomatis infections in infants. JAMA 267:400-402, 1992.

28. Harrison HR, Phil D, Taussig LM, Fulginiti VA: Chlamydia trachomatis and chronic respiratory disease in childhood. Pediatr Infect Dis J 1:29-33, 1982. 


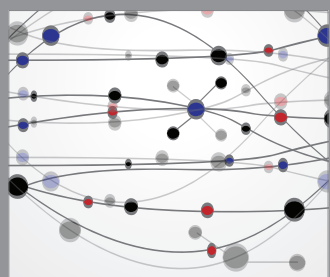

The Scientific World Journal
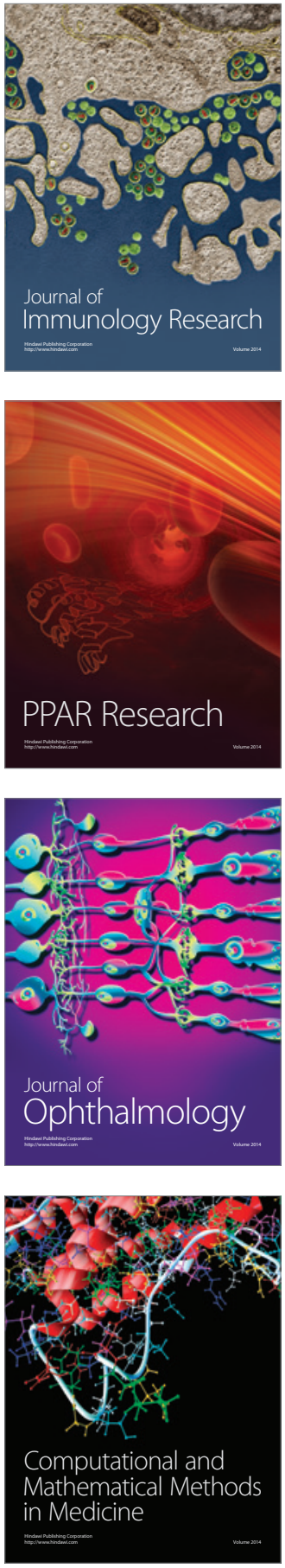

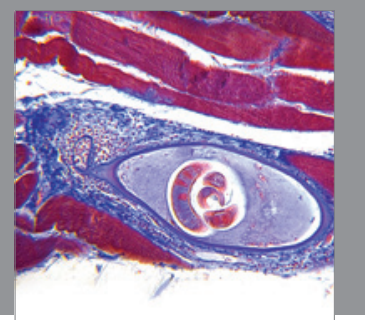

Gastroenterology

Research and Practice
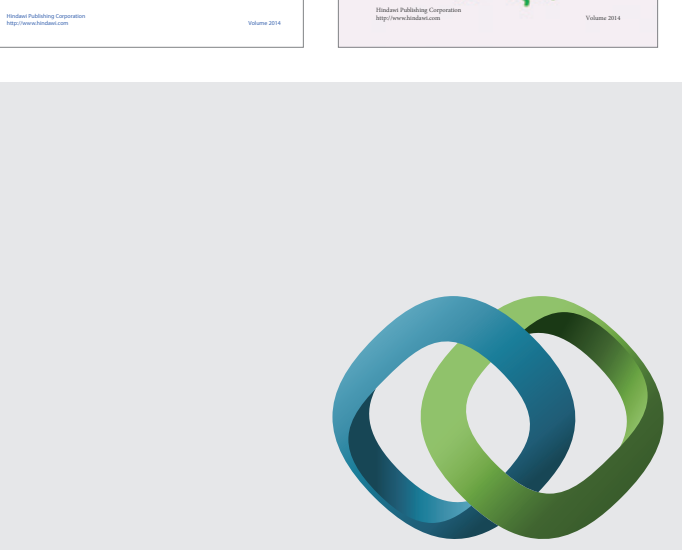

\section{Hindawi}

Submit your manuscripts at

http://www.hindawi.com
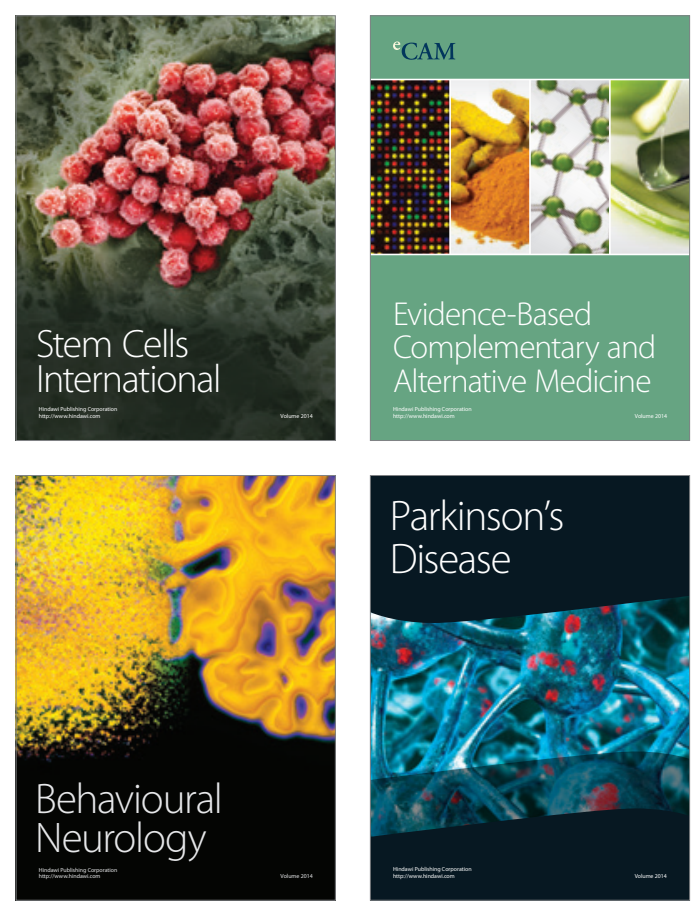

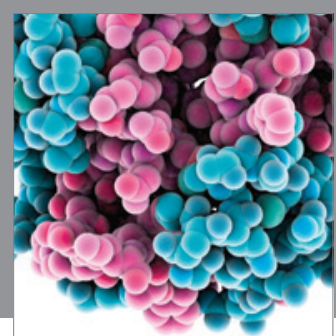

Journal of
Diabetes Research

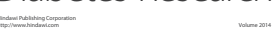

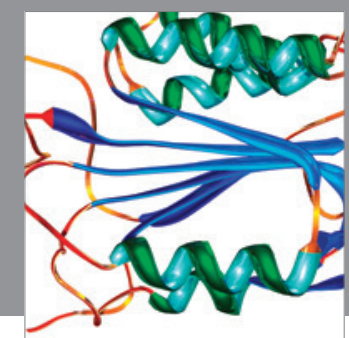

Disease Markers
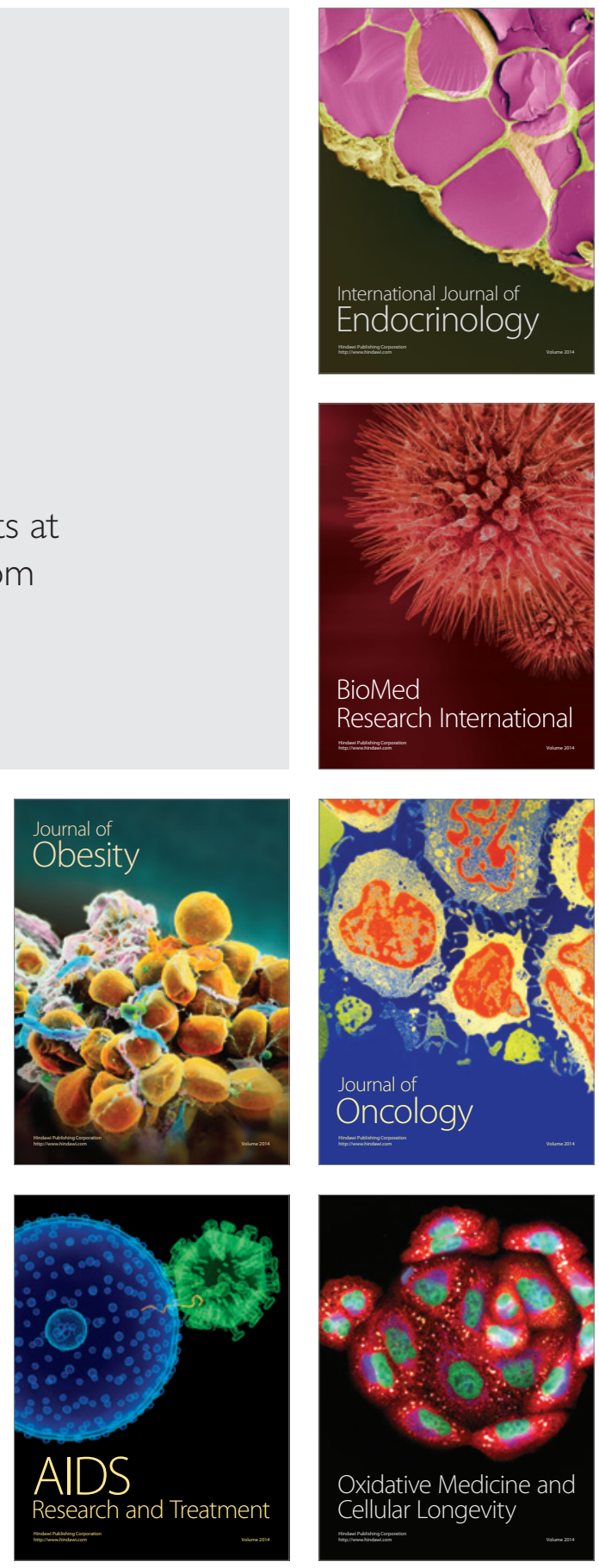